\title{
Factores psicosociales asociados a trastornos depresivos en pacientes del Hospital Central de Nampula, Mozambique.
}

\author{
Psychosocial factors associated with depressive disorders in patients at Nampula Central Hospital, \\ Mozambique.
}

Andrés Vázquez Machado ${ }^{1, a,}$, Julienne Mukamutara ${ }^{2, b}$

\section{RESUMEN}

Objetivo: Identificar factores psicosociales claramente asociados a depresión en una población susceptible. Material y Métodos: Estudio transversal en 347 pacientes con diagnóstico de depresión y 168 sin trastornos mentales ni síntomas psicológicos actuales, atendidos en un hospital de Mozambique, entre febrero de 2014 y enero de 2017. Se estudiaron variables socio-demográficas así como algunos eventos adversos tempranos y eventos vitales actuales. Se condujeron análisis bivariados, utilizando chi cuadrado y odds ratio. Resultados: Se encontró una asociación significativa $(\mathrm{p}<0,05)$ entre los trastornos depresivos y factores socio-demográficos como 34 años de edad o menos, ser divorciado o soltero, desempleado y tener entre ninguno y dos hijos. El promedio de eventos adversos fue de 2,4 entre los deprimidos y 0,6 entre los no deprimidos. Eventos adversos (i.e., violencia intrafamiliar y muerte de uno de los padres, entre los más frecuentes) durante la edad temprana se asociaron con depresión en la adultez, incrementando en 5 veces tal probabilidad, en tanto que la ocurrencia de 2 o más eventos en la etapa actual generó el mismo resultado. Los más comunes fueron los conflictos interpersonales y el padecimiento de una enfermedad física. Conclusiones: Experiencias adversas en la infancia y diversos eventos vitales actuales son más frecuentes en pacientes con trastornos depresivos que en pacientes sin depresión, confirmando su vigencia como factores psicosociales de riesgo.

PALABRAS CLAVE: Depresión, eventos vitales, factores psicosociales.

\section{SUMMARY}

Objective: To identify psychosocial factors associated with depression in a susceptible patient population. Material and Methods: Cross-sectional study in 347 depressed and 168 non-depressed patients who attended the Nampula Central Hospital, Mozambique, between February 2014 and November 2016. Sociodemographic variables as well as early and current adverse events were studied. The associations between all variables and depression were subjected to bivariate analyses with Chi square test and odds ratio test. Results: A significant association $(p<0,05)$ was found between depressive disorder and socio-demographic factors such as 34 years of age or less, single or divorced status, unemployment and having none to two children. The mean number of current adverse events in depressed

\footnotetext{
Policlínico Jimmy Hirzel, Bayamo. Granma, Cuba.

Hospital Central de Nampula. Nampula, Mozambique.

Médico-Psiquiatra.

Psicóloga.
} 
Factores psicosociales asociados a trastornos depresivos en pacientes del Hospital Central de Nampula, Mozambique.

patients was 2.4, and 0.6 in those non-depressed. Early adverse events such as interparental violence and the death of one of the parents were associated with adult depression in adulthood and a 5-fold increase in the likelihood of experiencing depression, while experiencing 2 or more current events in the current time resulted in the same. The most frequent were interpersonal conflicts and suffering from a physical illness. Conclusions: Adverse childhood experiences and a variety of current life events occur more frequently in patients with depressive disorders than in patients without depression, thus confirming its role as psychosocial risk factors.

KEYWORDS: Stressful life events, psychosocial factors, depression.

\section{INTRODUCCIÓN}

Han pasado 398 años desde que en 1621 Robert Burton publicara su obra Anatomía de la Melancolía, en el cual ofrecía una concepción multifactorial de la depresión. En la actualidad esta enfermedad se ha convertido en uno de los principales problemas de salud de la humanidad y según estimados de la Organización Mundial de la Salud se convertirá en la segunda causa de discapacidad en los próximos años, después de las enfermedades cardiovasculares (1). A pesar de que el desarrollo científico técnico ha permitido avances en el conocimiento de la depresión, aún continúa siendo impreciso el conocimiento de las causas que la provocan y persiste la concepción multifactorial de la misma, invocándose factores biológicos, genéticos, psicológicos y sociales en su génesis (2).

La asociación entre los factores psicosociales y la depresión es motivo de estudio de múltiples investigadores. Se destacan diversos eventos psicotraumatizantes que afectan la persona en el curso de su vida, que alteran el mecanismo regulador del estrés y desencadenan cambios bioquímicos a nivel del Sistema Nervioso Central (SNC), perturban la secreción hormonal del eje hipotálamo - hipófisis - adrenal (HHA), hipotálamo - hipófisis - tiroides y la liberación de hormonas sexuales; todos ellos implicados en los cambios del humor presentes en el trastorno (3). La alteración en la secreción de citocinas proinflamatorias, que da lugar a una activación de la reacción inflamatoria como respuesta a los estresores psicosociales constituye un mecanismo causal involucrado en la enfermedad (4).

Diversos hallazgos corroboran que los eventos psicotraumatizantes que acontecen en la infancia predisponen a la depresión en la edad adulta $(2,5)$. Los individuos que han experimentado eventos adversos tempranos (EAT) muestran una exagerada respuesta inflamatoria a estresores y ellos probablemente desarrollarán una depresión en meses posteriores $(4,6)$. Aproximadamente $1 / 4$ a $1 / 3$ de los niños maltratados van a reunir criterios para un trastorno depresivo mayor (TDM) cuando alcancen el final de la segunda década de su vida (7). Diversos estresores como el maltrato en la niñez se han asociado con la reducción del volumen del hipocampo en la adultez (8). También se han encontrado alteraciones en otras regiones cerebrales como la amígdala y la corteza prefrontal, las que están en dependencia del evento y del tiempo en que estuvo afectando al niño (9).

Los eventos vitales actuales (EVA) son aquellos acontecimientos que ocurren en fecha reciente y tienen un impacto negativo en el bienestar psicológico del individuo. Algunos estudios consideran los que acaecieron en los 6 meses previos $(5,10)$; aunque mayormente se tienen en cuenta los que ocurrieron en los últimos 12 meses $(2,11,12)$. Todavía es un área de debate los tipos de estresores que constituyen factores de riesgo de depresión y el rol que tienen como predictor de la enfermedad: son causantes del cuadro depresivo, agravan el curso, interfieren con el resultado del tratamiento o son responsables de las recaídas y recurrencia. Se ha señalado que su efecto varía de una población a otra (13).

El efecto depresogénico de las experiencias negativas es señalado en algunas investigaciones. Un metaanálisis de Risch $\mathrm{N}$ et al (14) que incluyó 14 estudios, demostró que los eventos estresantes de la vida tienen una fuerte asociación con el riesgo de deprimirse.

En el Hospital Central de Nampula (HCN) se atienden muchos pacientes con depresión; sin embargo, no existen estudios publicados en el país sobre la enfermedad, ni los factores relacionados con ella; por lo que este estudio constituye una novedad científica en ese sentido. 
El objetivo de esta investigación fue identificar algunos factores psicosociales asociados a la depresión en un grupo de pacientes con diversos trastornos depresivos.

\section{MATERIAL Y MÉTODOS}

Se realizó un estudio transversal, de serie de casos, con pacientes que asistieron al HCN, Mozambique, en el periodo comprendido entre febrero de 2014 y enero de 2017.

Se escogió una muestra no aleatoria que quedó constituida por 347 pacientes deprimidos, que aceptaron participar en la investigación, previo conocimiento del propósito de esta. Se excluyeron los que no hablaban portugués y los que tenían diagnóstico comórbido de esquizofrenia.

También se incluyeron 168 pacientes que no tenían antecedentes de enfermedad mental, atención psiquiátrica ni síntomas psicológicos actuales y que fueron atendidos por otras causas en dicho hospital.

Se estudiaron las siguientes variables:

Sociodemográficas: edad, sexo, estado conyugal, ocupación, escolaridad y número de hijos.

Trastornos depresivos: incluyó diversos subtipos de depresión: TDM, trastorno distímico, duelo patológico, depresión asociada a enfermedad médica, trastorno depresivo menor, depresión puerperal, trastornos de personalidad descompensado con cuadro depresivo, trastorno disfórico premenstrual, trastorno bipolar en fase depresiva, trastornos adaptativos con estado de ánimo depresivo y otros trastornos depresivos no especificados. El diagnóstico fue realizado, mediante entrevista por un especialista en psiquiatría debidamente capacitado (autor).

Eventos adversos tempranos: se exploró mediante la entrevista aquellos eventos psicotraumatizantes que acontecieron antes de los 15 años de edad. Se identificó el número y el tipo de eventos. Para fines de esta investigación se consideró la muerte de uno o ambos progenitores, violencia intrafamiliar, ser víctima de maltrato físico y/o de abuso sexual. Como abuso sexual se incluyó el abuso lascivo, intento de violación y la violación, siempre que fuera ejercida por adolescentes o adultos (conocidos o extraños).

Eventos vitales actuales: consiste en un listado de 14 eventos psicotraumatizantes que pueden ser experimentados en los 12 meses previos a la entrevista y que acontecen en el contexto familiar, laboral, social, interpersonal o individual. Se tuvo en cuenta el número y tipo de evento. La identificación de estos eventos se realizó mediante la entrevista.

Los pacientes se escogieron en las salas de hospitalización, consultas externas de diabetes, psiquiatría, y medicina general. Como parte de la entrevista que se realiza a todos los pacientes, se obtenían los elementos para hacer el diagnóstico de enfermedad depresiva, o descartar cualquier afección psíquica que permitiera asignarlo al grupo de controles. Posteriormente se informaba acerca de la investigación y se solicitaba su anuencia para participar en el estudio; cuando la respuesta era afirmativa se procedía a la aplicación del cuestionario.

Se usó un cuestionario estructurado aplicado por entrevista que recoge los datos relativos a Información sociodemográfica: edad, sexo, estado conyugal, ocupación, escolaridad y número de hijos.

Factores ambientales y circunstanciales del eje III de la CIE - 10: la misma contempla conflictos relacionados con el grupo primario de apoyo, problemas relacionados con hechos negativos en la infancia (son los EAT), enfermedades somáticas propias, problemas económicos y con la vivienda, legales, muerte o enfermedad dentro de la familia, soporte familiar inadecuado, viudez reciente y otros problemas familiares inespecíficos.

Se hizo una distribución porcentual de las variables sociodemográficas, los EAT y EVA. Además, se calculó media aritmética, razón y rangos para la variable edad. Luego se hicieron análisis bivariados para determinar la asociación de la depresión con las variables cuantitativas y las demás variables categóricas. Se utilizó la prueba Chi Cuadrado, con corrección de Yates y se consideró significativa la asociación que tuvo una probabilidad menor que 0,05 . Para determinar si las variables estudiadas se convierten en factores de riesgo de la depresión se calculó la razón de disparidad (odds ratio) con un intervalo de confianza de $95 \%$. Se empleó el paquete estadístico EPINFO versión 2002.

El estudio contó con la aprobación del Comité Científico (hace función de Comité de Ética) de la Institución. Previo a la realización de la investigación se solicitó el consentimiento oral para participar en el estudio. En el caso de los individuos menores de edad se contó con el consentimiento de uno de los padres. 
Factores psicosociales asociados a trastornos depresivos en pacientes del Hospital Central de Nampula, Mozambique.

Tabla 1. Características sociodemográficas de pacientes con y sin depresión.

\begin{tabular}{|c|c|c|c|c|}
\hline \multirow[t]{2}{*}{ Características demográficas } & \multicolumn{2}{|c|}{$\begin{array}{c}\text { Con depresión } \\
(n=347)\end{array}$} & \multicolumn{2}{|c|}{$\begin{array}{l}\text { Sin depresión } \\
(n=168)\end{array}$} \\
\hline & $n^{0}$ & $\%$ & $n^{0}$ & $\%$ \\
\hline \multicolumn{5}{|l|}{ Grupos de edades } \\
\hline 34 años o menos & 140 & 40,3 & 49 & 29,2 \\
\hline $35-54$ años & 152 & 43,8 & 85 & 50,6 \\
\hline 55 y más años & 55 & 15,9 & 34 & 20,2 \\
\hline \multicolumn{5}{|l|}{ Sexo } \\
\hline Femenino & 227 & 65,4 & 109 & 64,9 \\
\hline Masculino & 120 & 34,6 & 59 & 35,1 \\
\hline \multicolumn{5}{|l|}{ Estado conyugal } \\
\hline Con pareja & 209 & 60,2 & 131 & 78,0 \\
\hline Divorciado & 64 & 18,4 & 15 & 8,9 \\
\hline Soltero & 49 & 14,1 & 11 & 6,5 \\
\hline Viudo & 25 & 7,2 & 11 & 6,5 \\
\hline \multicolumn{5}{|l|}{ Ocupación } \\
\hline Doméstica & 103 & 29,7 & 51 & 30,4 \\
\hline Con empleo & 161 & 46,4 & 91 & 54,2 \\
\hline Desempleado & 41 & 11,8 & 9 & 5,4 \\
\hline Estudiante & 27 & 7,8 & 7 & 4,2 \\
\hline Jubilado & 15 & 4,3 & 10 & 5,9 \\
\hline \multicolumn{5}{|l|}{ Número de hijos } \\
\hline $0-2$ & 172 & 49,6 & 56 & 33,3 \\
\hline $3-4$ & 74 & 21,3 & 50 & 29,8 \\
\hline 5 o más & 101 & 29,1 & 62 & 36,9 \\
\hline
\end{tabular}

Tabla 2. Eventos vitales adversos en pacientes son y sin depresión.

\begin{tabular}{|c|c|c|c|c|}
\hline \multirow[t]{2}{*}{ Eventos adversos } & \multicolumn{2}{|c|}{$\begin{array}{c}\text { Con depresión } \\
(n=347)\end{array}$} & \multicolumn{2}{|c|}{$\begin{array}{c}\text { Sin depresión } \\
\quad(n=168)\end{array}$} \\
\hline & $n^{0}$ & $\%$ & $n^{0}$ & $\%$ \\
\hline \multicolumn{5}{|l|}{ Eventos adversos tempranos* } \\
\hline Violencia intrafamiliar & 91 & 26,3 & 4 & 2,4 \\
\hline Muerte de uno de los padres & 90 & 26,0 & 21 & 12,5 \\
\hline Maltrato físico & 72 & 20,7 & 7 & 4,2 \\
\hline Abuso sexual & 4 & 12,7 & 4 & 2,4 \\
\hline \multicolumn{5}{|l|}{ Eventos vitales actuales } \\
\hline Conflictos con la pareja & 125 & 36,0 & 9 & 5,4 \\
\hline Enfermedad física & 96 & 27,7 & 16 & 9,5 \\
\hline Víctima de maltrato: & 95 & 27,4 & 1 & 0,6 \\
\hline Conflicto con la familia & 87 & 25,1 & 8 & 4,8 \\
\hline Muerte de un familiar querido & 81 & 23,3 & 39 & 23,2 \\
\hline Dificultades económicas & 71 & 20,5 & 4 & 2,4 \\
\hline Separación o divorcio & 67 & 19,3 & 6 & 3,6 \\
\hline Problemas con la vivienda & 27 & 7,8 & - & - \\
\hline Problemas en el trabajo & 27 & 7,8 & - & - \\
\hline Familiar enfermo & 23 & 6,6 & 16 & 9,5 \\
\hline Problemas legales & 11 & 3,2 & - & - \\
\hline Robo & 7 & 2,0 & - & - \\
\hline
\end{tabular}


Se utilizaron los datos obtenidos solo con fines de la investigación, cuidando la privacidad requerida en estos casos.

\section{RESULTADOS}

Se entrevistaron a 347 pacientes con depresión, con un promedio de edad de 39,6 años (rango: 14 - 79 años). Las mujeres representaron $65,4 \%$ de la muestra; razón mujer/hombre: 1,9. El mayor porcentaje correspondió a los que tenían pareja, un empleo, escolaridad preuniversitaria y 1 o 2 hijos, o ninguno (tabla 1).

Los EAT fueron explorados en 97,4 \% de los pacientes con depresión. De ellos 54,2\% presentaron alguna adversidad. Entre los que no tenían depresión solo $19,6 \%$ experimentaron algún evento adverso en su infancia. Los más frecuentes fueron la violencia intrafamiliar y la muerte de uno de los padres (tabla 2). La mayoría de los pacientes con depresión $(95,7 \%)$ experimentaron uno o más EVA en los 12 meses previos a la entrevista. Entre los que no tenían depresión solo $53 \%$ mencionó que fue afectado por estos eventos. El promedio de EVA fue de 2,4 entre los pacientes con depresión y 0,6 entre los que no tenían depresión. Los eventos más frecuentes fueron los conflictos interpersonales, padecer una enfermedad física y ser víctima de maltrato físico, psicológico y/o sexual (tabla 2).

Al realizar análisis bivariados se encontró que se asociaron significativamente con la depresión $(p<0,05)$ la edad de 34 años o menos, el estatus de divorciado o soltero, estar desempleado, tener $1 \mathrm{o}$ 2 hijos, o ninguno, experimentar eventos adversos en la infancia y 2 o más eventos vitales en el último año. Experimentar EAT incrementó en 5 veces la probabilidad de padecer depresión en la edad adulta. Los pacientes que experimentaron 2 EVA tuvieron 6,62 veces más probabilidades de estar deprimidos, la que se incrementó a 43,38 cuando la cantidad de eventos eran 3 o más (tabla 3 ).

\section{DISCUSIÓN}

Los resultados de este estudio confirman la relación de algunas variables sociodemográficas con la presencia de trastornos depresivos en esta población. Las personas con depresión son jóvenes que están en la etapa más productiva de la vida, con predominio de las mujeres. Los hallazgos de otros autores difieren en la edad en que prevalecen los trastornos depresivos, reportando que la frecuencia más alta se encuentra en grupos de edades mayores a la de este estudio $(2,11,13,15)$. Sin embargo, existe coincidencia entre otras investigaciones y esta en cuanto a la relación del estatus conyugal y la ocupación con la enfermedad. Varios investigadores señalan que las personas que no tienen pareja son más propensas a deprimirse que las que tienen una pareja estable $(12,16,17)$. La ocupación provee a la persona de recursos económicos, salud física y salud mental, por lo que no tener empleo se convierte en un estresor que puede desencadenar alteraciones del estado de ánimo. Cuando se pierde el empleo se afectan el ingreso económico, el estatus y las

Tabla 3. Factores asociados con trastornos depresivos.

\begin{tabular}{|c|c|c|c|c|c|c|}
\hline & $\%$ & $\chi^{2}$ & p & OR & $\begin{array}{c}\text { L.I } \\
\text { IC: } 95 \%\end{array}$ & $\begin{array}{c}\text { L.S } \\
\text { IC: } 95 \%\end{array}$ \\
\hline \multicolumn{7}{|l|}{ Grupos de edades } \\
\hline 34 años o menos & 40,3 & 6,0777 & 0,012 & 1,64 & 1,10 & 2,44 \\
\hline \multicolumn{7}{|l|}{ Estado conyugal } \\
\hline Divorciado & 18,4 & 7,8764 & 0,005 & 2,31 & 1,27 & 4,18 \\
\hline Soltero & 14,1 & 6,2954 & 0,012 & 2,35 & 1,19 & 4,64 \\
\hline \multicolumn{7}{|l|}{ Ocupación } \\
\hline Desempleado & 11,8 & 5,3757 & 0,020 & 2,37 & 1,12 & 4,99 \\
\hline Estudiante & 7,8 & 2,3935 & 0,121 & 1,94 & 0,83 & 4,55 \\
\hline \multicolumn{7}{|l|}{ Número de hijos } \\
\hline $0-2$ & 49,6 & 12,0686 & 0,000 & 1,97 & 1,34 & 2,88 \\
\hline \multicolumn{7}{|l|}{ Eventos adversos } \\
\hline EAT* & 54,2 & 58,9339 & 0,000 & 5,13 & 3,31 & 7,94 \\
\hline $2 \mathrm{EVA}$ & 31,7 & 39,7639 & 0,000 & 6,62 & 3,45 & 12,71 \\
\hline 3 o más EVA & 44,7 & 93,9561 & 0,000 & 43,38 & 13,58 & 138,55 \\
\hline
\end{tabular}


Factores psicosociales asociados a trastornos depresivos en pacientes del Hospital Central de Nampula, Mozambique.

relaciones sociales, a lo que se puede añadir el estrés relacionado con el estigma por estar desempleado $(2,18,19)$.

Otro resultado que contradice lo reportado en la literatura es en cuanto al número de descendientes, ya que tener dos, uno, o ninguno se asoció con la presencia de trastornos depresivos; a pesar de que se conoce que tener muchos hijos aumenta el estrés por la sobrecarga que representa atender sus necesidades de estudios, cuidados, salud y alimentación, la afectación económica y convivencia. Una explicación para este resultado es que en la población de Nampula tener 3 o más hijos es algo común, cuando la persona tiene 2 o menos puede ser reflejo de algún evento que impida alcanzar un mayor número ej. tener VIH/SIDA puede limitar la cantidad por el riesgo de transmisión vertical y en este caso se convierte en un factor generador de estrés. La expectativa de un segmento poblacional amplio es tener varios hijos, y una de las explicaciones que dan las personas es que la posibilidad de muerte por enfermedades que son comunes en Mozambique (malaria, SIDA, dengue, cólera, entre otras) es muy alto, por lo tanto, tener una descendencia amplia garantiza no quedarse sin ninguno en caso de morir uno o varios de ellos.

Experimentar EAT, de manera general, se asocia con la presencia de depresión en la adultez. De acuerdo con la teoría cognitiva de la depresión, ser afectado por adversidades en la infancia provoca pensamientos disfuncionales y una interpretación distorsionada de la realidad, relacionado con la creencia errónea: "si mi vida empezó mal, va a estar mal siempre". Este sesgo cognitivo puede ser responsable también del estilo atribucional del deprimido, mediante el cual atribuye los sucesos que lo afectan a "su incapacidad para lograr que alguna cosa le salga bien", considerándolo como algo que inevitablemente le ocurrirá siempre y que permeabilizará todas las esferas de su vida. Esta vulnerabilidad cognitiva se convierte en un factor de riesgo de depresión y es responsable también de una mayor susceptibilidad al efecto depresogénico de las adversidades que se le presentan en la adultez y de un estilo interpersonal generador de dificultades en la adaptación social y de respuestas depresivas ante conflictos interpersonales (20).

Estos eventos que afectan a la persona en etapas tempranas también lo condicionan a tener una reacción exagerada al estrés, relacionado probablemente con alteraciones anatómicas a nivel del SNC y anormalidades en el funcionamiento del eje $\operatorname{HHA}(3,7-$
9). Por otro lado, se le brinda importancia al llamado estrés crónico que es resultado de estas experiencias adversas en los inicios de la vida con una activación excesiva y prolongada del sistema de respuesta al estrés en ausencia de factores protectores, el cual tiene un efecto deletéreo sobre el cerebro provocando las alteraciones estructurales, con las consecuentes dificultades en el neurodesarrollo; así como daño social, emocional y cognitivo (21).

Otras investigaciones informan resultados diferentes al de este estudio. Vitriol y colaboradores encontraron un porcentaje mayor de EAT en una muestra de dos ciudades de la Región del Maule, Chile, donde $82 \%$ de los pacientes con depresión presentaron al menos un EAT (5). Un estudio similar en Llallagua, Bolivia, también encontró una proporción de pacientes con depresión que experimentaron EAT superior al de este estudio (2). En una amplia muestra de pacientes atendidos en Clínicas de Atención Primaria en Calgary, Canadá, $70 \%$ de los deprimidos había experimentado al menos un EAT; en este estudio participaron pacientes con trastorno depresivo mayor, el diagnóstico se realizó empleando una escala de síntomas depresivos (PHQ-9) y se exploraron 10 tipos de EAT, incluyendo en el instrumento otros tipos de maltrato y de disfunción familiar (15).

Las diferencias con otros estudios pueden ser resultado de factores individuales como son: sesgos de memoria, resistencia a evocar sucesos dolorosos que ocurrieron hace muchos años (sobre todo si existen EVA a los cuales el sujeto le atañe la causa de su depresión) y la valoración actual que hace el individuo de un suceso que aconteció en su infancia. También el tipo y el número de eventos explorados, las características generales de los estudios y la forma de escoger las muestras. En la presente investigación, por tratarse de un estudio transversal, los sesgos de memoria pueden tener más relevancia, teniendo en cuenta que se trata de sujetos con depresión; además el hecho de que no se realizó un análisis multivariado limita la valoración que se hace de los resultados.

Björkenstam et al., (22) y Li M et al., (23) coinciden en afirmar que los EAT son predictores de síntomas depresivos y de diversos subtipos de depresión en los inicios de la edad adulta y etapas posteriores. Las personas que experimentan adversidades en su infancia tienen mayor probabilidad de tener una reacción exagerada al estrés que acaece en cualquier etapa de su vida (24). Los pacientes que estuvieron expuestos a EAT experimentan mayor 
cantidad de EVA cuando llegan a la adolescencia y esa susceptibilidad se mantiene en la edad adulta, determinado probablemente por un bajo umbral de tolerancia para eventos adversos o por la adopción de estilos conductuales y afectivos que se incorporan en la personalidad y los vuelven más sensibles a los eventos que se presentan en la vida (10).

La mayoría de los pacientes con depresión experimentaron algún EVA y la razón de disparidad informa que estos aumentan en casi 20 veces la probabilidad de deprimirse. Cuando se experimentan más de 2 eventos esa probabilidad se duplica. Este resultado permite confirmar la hipótesis de que existe una asociación positiva entre los EVA y el riesgo de depresión y que mientras mayor es la cantidad de EVA, mayor es la probabilidad de presentar un trastorno depresivo; lo cual coincide con investigaciones previas que también encontraron un alto número de EVA en pacientes deprimidos $(25,26)$. Soman et al., (27) informaron que los hombres experimentan como promedio 3,57 $\pm 2,43$ EVA y las mujeres $3,31 \pm 2,12$, siendo más frecuente los eventos dependientes y de significado negativo para ambos sexos. Los autores concluyen que el número total de eventos es alto para todos los deprimidos. You y Conner (28) reportaron que el número de eventos adversos severos (según la evaluación que hizo el entrevistado) se asoció significativamente con la presencia de síntomas depresivos actuales en adolescentes y adultos jóvenes que no tenían antecedentes de trastornos depresivos; el número de eventos leves y moderadamente estresantes se asoció con la sintomatología depresiva en ambos: los que no tenían antecedentes de depresión y los que tenían historia de la enfermedad.

Los conflictos interpersonales, que muchas veces generan violencia y son causa de separación o divorcio; así como las enfermedades físicas, son los eventos más frecuentes que experimentaron los deprimidos en el año previo a la entrevista. Los problemas con la pareja y la familia son los principales EVA en muestras clínicas, sobre todo para las mujeres; $(2,10,27,29)$ esos problemas afectan la convivencia, dañan la principal fuente de apoyo social del individuo y son generadores de estrés, autoestima baja, desesperanza y desamparo. La alta frecuencia con que se señala padecer una enfermedad física como evento estresante se explica porque una parte importante de la muestra se escogió en las salas de hospitalización y consultas externas donde se atienden pacientes con diabetes mellitus, VIH/SIDA, entre otras. La relación de la enfermedad física y la depresión es bidireccional; por una parte, algunas enfermedades causan depresión debido a su efecto sobre el SNC y el sistema neuroendocrino y por otra parte la enfermedad se convierte en un estresor psicosocial que perturba el bienestar psicológico del paciente, ya sea por las molestias propias de la dolencia, la discapacidad que ocasiona o la afectación económica por la incapacidad para trabajar y los gastos por tratamiento médico. Diversas investigaciones coinciden en señalar la enfermedad física como un EVA en pacientes deprimidos $(13,17,26)$.

Una limitación de este estudio es que a un número elevado de pacientes solo se les realizó una entrevista, lo cual puede reducir la posibilidad de informar más elementos relacionados con eventos adversos; también la forma de escoger la muestra y que solo se realizaron análisis bivariados limita la extrapolación de los resultados; por lo que se requieren nuevos estudios con muestras poblacionales aleatorizadas. $\mathrm{Su}$ principal aporte es apoyar la teoría psicosocial de la depresión, al corroborar que la exposición a eventos adversos durante la infancia y la adultez predispone a la presencia de trastornos depresivos, lo que sugiere la necesidad de tenerlos en cuenta a la hora de brindar atención a pacientes deprimidos.

Se concluye que las personas deprimidas son mayormente jóvenes y de sexo femenino, que experimentan más EAT que los que no padecen depresión; también tienden a informar mayor cantidad de EVA, propiciado probablemente por su estado de ánimo, que provoca que interpreten los acontecimientos que les ocurren (que pueden ser iguales a los que le ocurren a la mayoría de las personas) desde su óptica depresiva, deprimiéndose con facilidad y con escasos recursos psicológicos para manejarlos de forma adecuada.

Agradecimientos: Al profesor Juan Manuel Costa Fonseca, de la Dirección Provincial de Educación de Granma, por la revisión y corrección de estilo de redacción del manuscrito.

\section{Correspondencia:}

Andrés Vázquez Machado.

Edificio 22. Apto 2. Reparto Granma CP: 75100. Bayamo, Granma, Cuba.

Teléfono: 23431904. Móvil: 55072897.

Correo electrónico: anvaz@infomed.sld.cu

No existe conflicto de intereses

No hubo fuentes de financiación. Se hizo con recursos propios. 
Factores psicosociales asociados a trastornos depresivos en pacientes del Hospital Central de Nampula, Mozambique.

\section{REFERENCIAS BIBLIOGRÁFICAS}

1. World Health Organization. Depression. Ginebra: World Health Organization; 2016 (Citado en abril del 2016) Disponible en: www.who.int/mediacentre/ factsheets/fs369/en/index.html

2. Vázquez A. Los factores psicosociales y la depresión. MULTIMED.2016; 20 (3):0-0. (Citado en febrero del 2018) Disponible en: http://www.revmultimed.sld. cu/index.php/mtm/article/view/319

3. Vázquez A. Depresión. Diferencias de género. MULTIMED. 2013; 17 (3): 0-0. (Citado en febrero del 2018) Disponible en: http://www.revmultimed. sld.cu/index.php/mtm/article/view/202

4. Miller AH, Raison CL. The role of inflammation in depression: from evolutionary imperative to modern treatment target. Nat Rev Immunol. 2016; 16(1): $22-$ 34.

5. Vitriol V, Cancino A, Leiva-Bianchi M, Serrano C, Ballesteros S, Potthoff S, et al. Depresión adulta y experiencias infantiles adversas: evidencia de un subtipo depresivo complejo en consultantes de la atención primaria en Chile. Rev Med Chile. 2017; 145 (9): 1145-1153.doi: 10.4067/s0034-9887201700090 1145

6. De Punder K, Entringer S, Heim C, Deuter CE, Otte $\mathrm{C}$, Wingenfeld, et al. Inflammatory measures in depressed patients with and without a history of adverse childhood experiences. Front Psychiatry 2018; 9: 610. doi: 10.3389/fpsyt.2018.00610

7. Juruena MF, Von Werne Baes C, Castro Menezes I, Graeff FG. Early life stress in depressive patient: role of glucocorticoid and mineralocorticoid receptors and of hypothalamic-pituitary-adrenal axis activity. Curr Pharm Des. 2015;21(11):1369-78.

8. Chen MC, Hamilton JP, Gotlib IH. Decreased hippocampal volume in healthy girls at risk of depression. Arch Gen Psychiatry. 2010; 67 (3): 270-6.

9. van Bodegom M, Homberg JR, Henckens MJAG. Modulation of the Hypothalamic-Pituitary-Adrenal Axis by Early Life Stress Exposure. Front Cell Neurosci.2017;11:87. doi:10.3389/fncel.2017.00087.

10. Korkeila J, Vahtera J, Nabi H, Kivimäki M, Korkeila $\mathrm{K}$, Sumanen $\mathrm{M}$, et al. Childhood adversities, adulthood life events and depression. J Affect Disord. 2010; 17: 130-8.

11. Harkness KL, Theriault JE, Stewart JG, Bagby RM. Acute and chronic stress exposure predicts 1 -year recurrence in adult outpatients with residual depression symptoms following response to treatment. Depression \& Anxiety. 2014; 31: 1-8.

12. Lamers F, Beekman ATF, de Jonge P, Smit JH, Nolen WA, Penninx BWJH. One-year severity of depressive symptoms: Results from the NESDA study. Psychiatr Res. 2011; 190: 226-31.

13. Assari S, Lankarani MM. Stressful life events and risk of depression 25 years later: race and gender differences. Front Public Health. 2016; 4:49. doi: 10.3389/fpubh.2016.00049.

14. Risch N, Herrell R, Lehner T, Liang KY, Eaves L, Hoh J. Interaction between the serotonin transporter gene (5-HTTLPR), stressful life events, and risk of depression: A meta-analysis. JAMA. 2009; 301 (23): 2462-71.

15. Poole JC, Dobson K, Pusch D. Childhood adversity and adult depression: The protective role of psychological resilience. Child abuse \& neglect. 2017;64:89-100.

16. Bulloch AGM, Williams JVA, Lavorato DH, Patten $\mathrm{SB}$. The depression and marital status relationship is modified by both age and gender. J Affect Dis. 2017; 223: 65-68. Doi: 10.1016/j.jad.2017.06.007

17. Maciejewski PK, Prigerson HG, Mazure CM. Selfefficacy as a mediator between stressful life events and depressive symptoms. Br J Psychiatry. 2000; 176: 373-8.

18. McGee RE, Thompson NJ. Unemployment and depression among emerging adults in 12 states, behavioral risk factor surveillance system, 2010. Prev Chronic Dis. 2015; 12: 140451. Doi: 10.5888/ pcd12.140451

19. Shaik S, Rajkumar RP, Menon V, Sarkar S. Gender, life events, and depression: An exploratory study. Indian J Psychol Med. 2017; 39: 330 - 5. (Citado en diciembre del 2018) Disponible en: http://www.ijpm. info/text.asp?2017/39/3/330/207339

20. Liu RT. Childhood adversities and depression in adulthood: Current findings and future directions. Clin Psychol (New York). 2017; 24 (2): 140-53.

21. Kimple KS, Kansagra SM. Responding to adverse childhood experiences. N C Med J. 2018; 79 (2): 95-8.

22. Björkenstam E, Vinnerljung B, Hjern A. Impact of childhood adversities on depression in early adulthood: A longitudinal cohort study of 478,141 individuals in Sweden. J Affect Disord. 2017; 223: 95-100. doi: 10.1016/j.jad.2017.07.030.

23. Li M, D'Arcy C, Meng X. Maltreatment in childhood substantially increases the risk of adult depression and anxiety in prospective cohort studies: systematic review, meta-analysis, and proportional attributable fractions. Psychol Med. 2016; 46 (4): 717-30. doi: 10.1017/S0033291715002743

24. Shapero BG, Black SK, Liu RT, Klugman J, Bender RE, Abramson LY, et al. Stressful Life Events and Depression Symptoms: The Effect of Childhood Emotional Abuse on Stress Reactivity. J Clin Psychol. 2014; 70 (3): 209-223.

25. Kendler KS, Thornton LM, Prescott CA. Gender Differences in the rates of exposure to stressful life events and sensitivity to their depressogenic effects. Am J Psychiatry 2001; 158 (4): 587-93.

26. Gilman SE, Trinh NH, Smoller JW, Fava M, Murphy 
JM, Breslau J. Psychosocial stressors and the prognosis of major depression: a test of Axis IV. Psychol Med. 2013; 43 (2): 303-16.

27. Soman S, Bhat SM, Latha KS, Praharaj SK. Gender differences in perceived social support and stressful life events in depressed patients. East Asian Arch Pychiatr. 2016; 26: 22-29.

28. You S, Conner KR. Stressful life events and depressive symptoms: Influences of gender, event severity, and depression history. J Nerv Ment Dis. 2009; 197 (11): 829-833. doi:10.1097/NMD.0b013e3181be7841
29. Wu M, Li JC, Yu CQ, Chen YP, Lyu J, Guo Y, et al. Gender differences in stressful life events and depression in Chinese adults aged 30-79 years. Zh Liu Xihg Bing Xue Za Zhi. 2017; 38 (11): 1449-53.

Recibido: 24/03/2020

Aceptado: 23/11/2021 